\title{
The Stable Topological-hyperbolic Space Form Problem for Complete Manifolds of Finite Volume
}

\author{
F.T. Farrell ${ }^{\star}$ and W.C. Hsiang ${ }^{\star \star}$ \\ * Department of Mathematics University of Michigan, Ann Arbor, MI 48109/USA \\ * Department of Mathematics, Princeton University, Princeton, NJ 08544, USA \\ * Partially supported by NSF grant number MCS-7701124 \\ * Partially supported by NSF grant number GP $34324 X 1$
}

\section{§1. Introduction and Statement of Results}

About fifteen years ago, A. Borel posed the following conjecture. Let $M^{n}$ be a closed aspherical manifold, i.e., $\pi_{i} M^{n}=0$ for $i>1$. If $g: N^{n} \rightarrow M^{n}$ is a homotopy equivalence where $N^{n}$ is another manifold, then $g$ is homotopic to a homeomorphism. A stable version of this conjecture for $M^{n}$ a closed nonpositively curved manifold was verified in [4, Corollary B]. Namely, if $M^{n}$ is a closed non-positively curved manifold and $g: N^{n} \rightarrow M^{n}$ is a homotopy equivalence where $N^{n}$ is a manifold, then

$$
g \times \mathrm{id}: N^{n} \times \mathbb{R}^{3} \rightarrow M^{n} \times \mathbb{R}^{3}
$$

is homotopic to a homeomorphism.

In this note, we shall extend this result to the non-compact case. Precisely, we have the following result.

Theorem A. Let $M^{n}$ be a complete Riemannian manifold of finite volume and whose sectional curvatures are strictly negative and bounded away from 0 and $-\infty$, (e.g., a complete hyperbolic manifold of finite volume). Let $N^{n}$ be a manifold and let $\mathrm{g}: N^{n} \rightarrow M^{n}$ be a proper homotopy equivalence. Then,

$$
g \times \text { id : } N^{n} \times \mathbb{R}^{3} \rightarrow M^{n} \times \mathbb{R}^{3}
$$

is properly homotopic to a homeomorphism.

In fact, we shall prove a less transparently formulated but much stronger result, Theorem 3.1, in $\S 3$. In $\S 4$ we will deduce Theorem A from an addendum to Theorem 3.1. Moreover, we shall discuss various versions of the conjectures and their relationships in $\S 4$. In particular, we shall verify the so-called Novikov's conjecture for $\pi=\pi_{1} M^{n}$ where $M^{n}$ is as in Theorem A. It should be pointed out (as we shall do in §4) that it will be rather delicate to generalize the result further to locally symmetric spaces of higher rank. 
We wish to thank John Morgan, whose very valuable suggestions on how to improve an earlier version of this paper has led to the present one. In particular, we are very grateful that he pointed out an error in our original statement of Addendum 3.1.1.

\section{§. Structure of the Cusps}

Let $M^{n}$ be a complete Riemannian manifold of finite volume and whose sectional curvatures are strictly negative and bounded away from 0 and $-\infty$. $M^{n}$ is diffeomorphic to the interior of a compact manifold with boundary [8]. Hence it has a finite number of ends. An open collar neighborhood $C$ of an end is called a cusp (motivated by the hyperbolic terminology) and the map $\pi_{1}(C) \rightarrow \pi_{1}(M)$ induced by inclusion $C \subset M$ is injective [8]. Let $C_{1}, C_{2}, \ldots, C_{q}$ be cusps, one for each end of $M$, and $f_{i}: \tilde{M} \rightarrow \mathbb{R}$ be Busemann functions, where $\tilde{M} \rightarrow M$ is the universal cover of $M$, satisfying the following conditions [3], [8], where $\tilde{C}_{i}$ is a fixed lift of the univeral cover of $C_{i}$ to $\tilde{M}$.

(i) $f_{i}$ has no critical points;

(ii) for $x \in \tilde{M}$, the gradient flow line passing through $x$ is a geodesic;

(iii) $f_{i}$ is $\pi_{1} C_{i}$ equivariant;

(iv) when $C_{i}$ and $\tilde{C}_{i}$ are appropriately chosen, we may assume that $\tilde{C}_{i}$ $=f_{i}^{-1}(0, \infty)$ without loss of generality.

Note that

$$
f_{i}^{-1}[1, \infty) / \pi_{1} C_{i}
$$

is a closed collar neighborhood of the end corresponding to $C_{i}$. Let $B_{i}$ denote

$$
f_{i}^{-1}(1) / \pi_{1} C_{i}
$$

which is a codimension-one submanifold of $C_{i}$. We next compactify $\tilde{M}^{n}$ to the $n$-dimensional disc $\mathbb{D}^{n}$ as follows. Choose a base point $p \in \tilde{M}^{n}$ and an orthogonal framing at $p$ thus identifying $\mathbb{R}^{n}$ to $T_{p} \tilde{M}^{n}$. The exponential map

$$
\exp : \mathbb{R}^{n} \rightarrow \tilde{M}^{n}
$$

is a distance non-decreasing diffeomorphism if we give $\mathbb{R}^{n}$ the Euclidean metric. Compactify $\tilde{M}^{n}$ by adding an end-point to each geodesic ray emanating from $p$. Consequently, any geodesic in $\tilde{M}$ has two distinct endpoints in $\mathbb{D}^{n}$. The natural action of $\pi_{1} M^{n}$ on $\tilde{M}^{n}$ extends to $\mathbb{I D}^{n}$ [4].

Let us now relate $\tilde{C}_{i}$ to the compactification $\mathbb{D}^{n}$. There is a unique point $c_{i} \in \mathbb{D}^{n}$ satisfying the following conditions.

(i) If we follow the gradient flow of $f_{i}$ in the positive direction, then every point $x \in \tilde{M}^{n}$ ends at $c_{i}$.

(ii) $f_{i}$ defines a product structure on $\mathbb{D}^{n}-c_{i}$ so that the quotient, under the action of $\pi_{1} C_{i}$, is $B_{i} \times[-\infty, \infty)$. And $C_{i}=B_{i} \times(0, \infty)$. 
For example, if $M^{n}$ is a hyperbolic manifold, then $\hat{C}_{i}$ is just a horoball tangent to the fixed point $c_{i} \in \partial \mathbb{D}^{n}$ of the action of the parabolic subgroup $\pi_{1} C_{i}$. (See Fig. 1.)

We next recall a very important theorem of Margulis-Gromov [8] concerning $\pi_{1} C_{i}$. Namely, there is a nilpotent normal subgroup of finite index in $\pi_{1} C_{i}$; i.e., there is an exact sequence

$$
1 \rightarrow K \rightarrow \pi_{1} C_{i} \rightarrow G \rightarrow 1
$$

where $G$ is a finite group and $K$ is a nilpotent subgroup of $\pi_{1} C_{i}$.

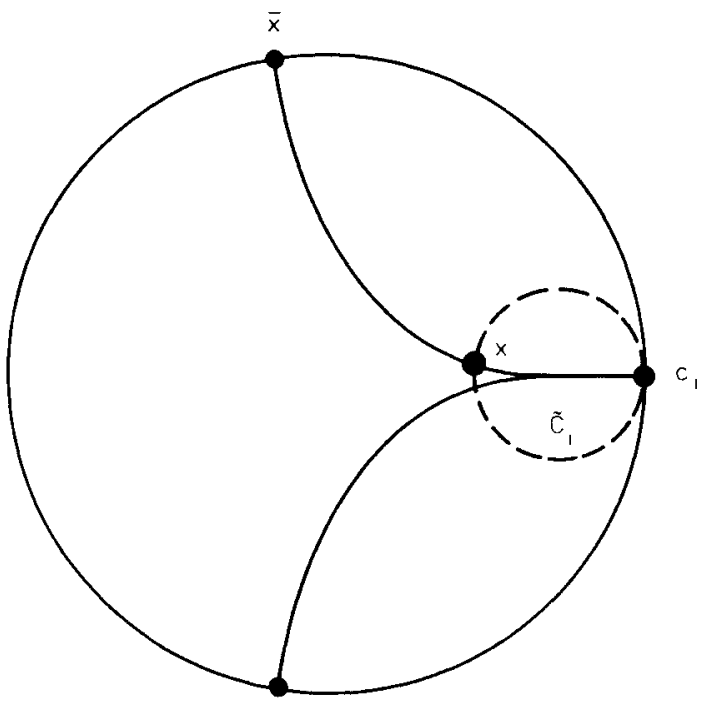

Fig. 1

Now, suppose that we have a proper homotopy equivalence

$$
\mathrm{g}: N^{n} \times \mathbb{D}^{k} \rightarrow M^{n} \times \mathbb{D}^{k} \quad(k \geqq 0)
$$

such that the restriction of $g$ to $N^{n} \times \partial \mathrm{D}^{k}$ is a homeomorphism. Consider the ends $g^{-1}\left(C_{i} \times \mathbb{D}^{k}\right)$ of $N^{n} \times \mathbb{D}^{k}$ corresponding to $C_{i} \times \mathbb{D}^{k}(i=1,2, \ldots, q)$.

Lemma 2.1. For $n+k \geqq 6$, we may assume that $g: N^{n} \times \mathbb{D}^{k} \rightarrow M^{n} \times \mathbb{D}^{k}$, after a proper homotopy, relative to $N^{n} \times \partial \mathbb{D}^{k}$, is a homeomorphism when restricted to $\mathrm{g}^{-1}\left(C_{i} \times \mathbb{D}^{k}\right)(i=1,2, \ldots, q)$.

Proof. Since $g$ is a proper homotopy equivalence, $g^{-1}\left(C_{i} \times \mathbb{D}^{k}\right)(i=1,2, \ldots, q)$ are tame ends with fundamental groups isomorphic to $\pi_{1} C_{i}$. It follows from [5] that we can produce codimension-one submanifolds $A_{i} \subset g^{-1}\left(C_{i} \times \mathbb{D}^{k}\right)$ 
which bound collar neighborhoods of infinity $A_{i} \times[1, \infty) \subset \mathrm{g}^{-1}\left(C_{i} \times \mathbb{D}^{k}\right)$ such that after a proper homotopy, relative to $N \times \partial \mathbf{D}$,

$$
\mathrm{g}: A_{i} \times[1, \infty) \rightarrow B_{i} \times[1, \infty) \times \mathbb{D}^{k}
$$

is a proper homotopy equivalence. In particular, $g: A_{i} \rightarrow B_{i} \times \mathbb{D}^{k}$ is a homotopy equivalence rel $\partial A_{i}$. Then, it follows from [6] that $g: A_{i} \rightarrow B_{i} \times \mathbb{D}^{k}$ is homotopic to a homeomorphism rel $\partial A_{i}$. By another proper homotopy, relative to $N \times \partial \mathrm{D}$, we produce $g$ as required.

Addendum 2.1.1. If $n+k \leqq 2$, the lemma is trivially true. If $3 \leqq n+k<6$, the conclusion of the lemma holds for $g^{\prime}: N^{n} \times \mathbb{D}^{k} \times T^{3} \rightarrow M^{n} \times \mathbb{D}^{k} \times T^{3}$ where $g^{\prime}$ is $g$ $\times \mathrm{id}_{T^{3}}$ and $T^{3}$ denotes the three-dimensional torus $S^{1} \times S^{1} \times S^{1}$.

Let us now consider the codimension- 0 submanifold $M_{1}^{n}$ of $M^{n}$ defined by

$M_{1}^{n}=M^{n}-\bigcup_{i=1}^{q} B_{i} \times(1, \infty)$.

Assume that $k \geqq 1$ and that $N^{n} \times \mathbb{D}^{k}$ is just another copy of $M^{n} \times \mathbb{D}^{k}$ (denoting the corresponding $M_{1}^{n}$ in $N^{n}$ by $N_{1}^{n}$ ) but that $g: N^{n} \times \mathbb{D}^{k} \rightarrow M^{n} \times \mathbb{D}^{k}$ is only a proper homotopy equivalence satisfying the following conditions:

(i) $g:\left(\overline{N^{n}-N_{1}^{n}}\right) \times \mathbb{D}^{k} \rightarrow\left(\overline{M^{n}-M_{1}^{n}}\right) \times \mathbb{D}^{k}$

is the identity homeomorphism;

(ii) $g^{-1}\left(M_{1}^{n} \times \mathbb{D}^{k}\right)=N_{1}^{n} \times \mathbb{D}^{k}$;

(iii) the restriction of $g$ to $N^{n} \times \partial_{-} \mathbb{D}^{k}$ is the identity map and the restriction of $g$ to $N \times \partial_{+} \mathbb{D}^{k}$ is a homeomorphism where $\partial_{+} \mathbb{D}^{k}$ and $\partial_{-} \mathbb{D}^{k}$

denote the upper and lower hemispheres of $S^{k-1}=\partial \mathbb{D}^{k}$, respectively.

So, $g$ induces a homotopy equivalence

$$
\mathrm{g}_{1}: N_{1}^{n} \times \mathbb{D}^{k} \rightarrow M_{1}^{n} \times \mathbb{D}^{k}
$$

such that $g_{1}$ restricted to $\partial\left(N_{1}^{n} \times \mathbb{D}^{k}\right)$ is a homeomorphism.

Let us now consider the compactification of $\tilde{M}^{n} \times \mathbb{D}^{k}$ (and of $\tilde{N}^{n} \times \mathbb{D}^{k}$ ) given as follows. View $\tilde{M}^{n}$ as the interior of $\mathbb{D D}^{n}$ and $S^{n-1}$ as the boundary of $\mathbb{D}^{n}$. Project $\tilde{M}^{n} \times \mathbb{D}^{k}$ to $\tilde{M}^{n}$ and shrink the size of $y \times \mathbb{D}^{k}$ (where $y \in \tilde{M}^{n}$ ) as $y$ moves to $\partial \mathbb{D}^{k}$ (and becomes a point as it gets to $\partial \mathbb{D}^{n}=S^{n-1}$ ). So, $\tilde{M}^{n} \times \mathbb{D}^{k}$ is compactified as the join $S^{n-1} * \mathbb{D}^{k}$ such that the action of $\pi_{1} M^{n}$ on $\tilde{M}^{n} \times \mathbb{D}^{k}$ via the first factor extends to $S^{n-1} * \mathbb{D}^{k}$.

Let us now fix a cusp $C_{i}$ of $M^{n}$ and a lifting $\tilde{C}_{i}$ of the universal cover of $C_{i}$ to $\tilde{M}^{n}$. Inside of $\tilde{C}_{i}$, we have $\tilde{B}_{i} \times(1, \infty)$ where $\tilde{B}_{i}$ denotes the lifting of the universal cover of $B_{i}$. Note that the point $c_{i}$ of $\mathbb{D D}^{n}$ is a point of $S^{n-1} \subset S^{n-1} * \mathbb{D}^{k}$. Let us consider the set $\tilde{L}_{i}$ defined by

$$
\tilde{L}_{i}=S^{n-1} * \mathbb{D}^{k}-\left[\tilde{B}_{i} \times(1, \infty) \times \mathbb{D}^{k} \cup c_{i}\right] .
$$

(See Fig. 2.) 


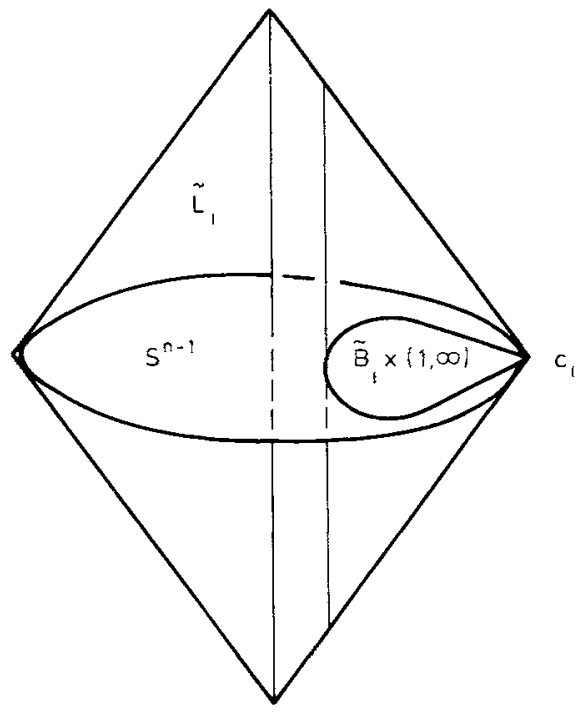

Fig. 2

Note that $\tilde{L}_{i}$ is a manifold homeomorphic to $\tilde{B}_{i} \times \mathbb{D}^{k+1}$ and $\pi_{1} C_{i}$ acts on $\tilde{L}_{i}$. The 'canonical' lift of $g$ to a map $\tilde{N}^{n} \times \mathbb{D}^{k} \rightarrow \tilde{M}^{n} \times \mathbb{D}^{k}$ extends uniquely to a self-map $\tilde{g}$ of $S^{n-1} * \mathbb{D}^{k}$ [cf. 4] satisfying

(i) $\tilde{g}^{-1}\left(\tilde{L}_{i}\right)=\tilde{L}_{i}$;

(ii) the restriction of $\tilde{\mathrm{g}}$ to $\left(\tilde{B}_{i} \times[1, \infty) \times \mathbb{D}^{k}\right) \cup\left\{c_{i}\right\}$ is the identity homeomorphism;

(iii) $\tilde{g} \mid S^{n-1} * \partial \mathbb{D}^{k}$ is a homeomorphism;

(iv) $\tilde{g} \mid S^{n-1} * \partial_{-} \mathbb{D}^{k}$ is the identity homeomorphism;

(v) denoting the restriction of $\tilde{\mathrm{g}}$ to $\tilde{L}_{i}$ by $\tilde{\mathrm{g}}_{i}, \tilde{\mathrm{g}}_{i}$ is a $\pi_{1} C_{i}$-equivariant proper self-homotopy equivalence of $\tilde{L}_{i}$;

(vi) $\tilde{g}_{i} \mid \partial \tilde{L}_{i}$ is a homeomorphism.

The homeomorphism (mentioned above) of $\tilde{L}_{i}$ to $\tilde{B}_{i} \times \mathbb{D}^{k+1}$ can be chosen so that $\tilde{B}_{i} \times \partial_{+} \mathbb{D}^{k+1}$ is identified with

$$
\tilde{L}_{i} \cap\left(S^{n-1} * \partial_{+} \mathbb{D}^{k}\right)
$$

hence denote this set by $\partial_{+} \tilde{L}_{i}$. Also, $\tilde{B}_{i} \times \partial_{-} \mathbb{D}^{k+1}$ is identified with

$$
\tilde{L}_{i} \cap\left(S^{n-1} * \partial_{-} \mathbb{D}^{k} \cup \tilde{B}_{i} \times 1 \times \mathbb{D}^{k}\right) ;
$$

hence denote this set by $\partial_{-} \tilde{L}_{i}$.

By (2.11.v), $\tilde{g}_{i}$ induces a self-homotopy equivalence $g_{i}: L_{i} \rightarrow L_{i}$ where the compact manifold $L_{i}$ is defined by

$$
L_{i}=\tilde{L}_{i} / \pi_{1} C_{i} .
$$


Note that $L_{i}$ is homeomorphic to $B_{i} \times \mathbb{D}^{k+1}$ and let $\partial_{+} L_{i}$ and $\partial_{-} L_{i}$ be defined by

(i) $\partial_{+} L_{i}=\partial_{+} \tilde{L}_{i} / \pi_{1} C_{i}$;

(ii) $\partial_{-} L_{i}=\partial_{-} \tilde{L}_{i} / \pi_{1} C_{i}$.

Also, $g_{i}$ satisfies

(i) $g_{i} \mid \partial L_{i}$ is a homeomorphism;

(ii) $g_{i} \mid \partial_{-} L_{i}=i d$.

Lemma 2.2. There is a continuous map $H: L_{i} \times[0,1] \rightarrow L_{i} \times[0,1]$ (provided $n$ $+k \geqq 6)$ satisfying

(i) $H(x, 0)=\left(g_{i}(x), 0\right)$ for all $x \in L_{i}$;

(ii) $H(x, 1)=(x, 1)$ for all $x \in L_{i}$;

(iii) $H^{-1}\left(L_{i} \times[0,1]\right)=L_{i} \times[0,1]$;

(iv) $H \mid \partial L_{i} \times[0,1]$ is a homeomorphism (concordance) onto $\partial L_{i} \times[0,1]$;

(v) $H \mid \partial_{-} L_{i} \times[0,1]$ is the identity homeomorphism.

Proof. Since $\pi_{1} C_{i}$ is finitely generated and torsion-free and contains a nilpotent subgroup of finite index, this lemma follows from [6] where we showed $\mathscr{S}\left(B_{i}\right.$ $\left.\times \mathbb{D}^{k+1}, \partial\right)=0$. (Recall if $M^{n}$ is a compact manifold with boundary $\partial M(n>4)$, then $\mathscr{S}(M, \partial)$ is an abelian group whose elements are equivalence classes of maps $f:\left(N^{n}, \partial N\right) \rightarrow\left(M^{n}, \partial M\right)$ where $N$ is a compact manifold and $f \mid \partial N$ is a homeomorphism onto $\partial M$. A second map $g:\left(K^{n}, \partial K\right) \rightarrow\left(M^{n}, \partial M\right)$ is equivalent to $f$ provided there is a homeomorphism $k:\left(K^{n}, \partial K\right) \rightarrow\left(N^{n}, \partial N\right)$ such that $f \circ k$ is homotopic to $g$ relative to $\partial K$. See [13, p. 247] and [14] for more details. More explicitly by the above identifications, $g_{i}$ determines an element of $\mathscr{S}\left(B_{i}\right.$ $\left.\times \mathbb{D}^{k+1}, \hat{\partial}\right)$ where $B_{i}$ is an aspherical manifold with virtually nilpotent fundamental group. But, by [6], $\mathscr{S}\left(B_{i} \times \mathbb{D}^{k+1}, \partial\right)=0$; hence $g_{i} \mid \partial L_{i}$ extends to a selfhomeomorphism of $L_{i}$; consequently $g_{i}\left|\partial_{+} L_{i}=g_{i}\right| B_{i} \times \partial_{+} \mathbb{D}^{k+1}$ is concordant, relative to $g_{i} \mid B_{i} \times \partial\left(\partial_{+} \mathbb{D}^{k+1}\right)$, to the identity homeomorphism. Use this concordance to define $H \mid \partial L_{i} \times[0,1]$ (so as to satisfy (iv) and (v) in particular). Then $H \mid\left(L_{i} \times 0 \cup \partial L_{i} \times[0,1]\right)$ determines another element of $\mathscr{S}\left(B_{i} \times \mathbb{D}^{k+1}, \partial\right)$ since $L_{i} \times 0 \cup \partial L_{i} \times[0,1]$ is homeomorphic to $B_{i} \times \mathbb{D}^{k+1}$. By a second application of $\mathscr{S}\left(B_{i} \times \mathbb{D}^{k+1}, \partial\right)=0, H$ extends to $L_{i} \times[0,1]$ satisfying (i)-(v).

Lemma 2.2 is the basis of the proof, given in $\S 3$, of the main result of this paper Theorem 3.1 .

\section{§3. Splitting the Surgery Exact Sequence}

Let $M_{1}^{n}=M^{n}-\bigcup_{i=1}^{q} B_{i} \times(1, \infty)$ be the manifold with the cusps cut off as introduced in $\S 2$. Then $M_{1}^{n}$ is a manifold with boundary. Consider the surgery exact sequence

$$
\begin{aligned}
& \stackrel{\theta}{\rightarrow} L_{n+k+1}^{s}\left(\pi_{1} M_{1}^{n}, \omega_{1}\left(M_{1}^{n}\right)\right) \\
& \rightarrow \mathscr{P}\left(M_{1}^{n} \times \mathbb{D}^{k}, \partial\right) \\
& \rightarrow\left[M_{1}^{n} \times \mathbb{D}^{k}, \partial ; G / \mathrm{Top}, *\right] \\
& \stackrel{\ominus}{\rightarrow} L_{n+k}^{s}\left(\pi_{1} M_{1}^{n}, \omega_{1}\left(M_{1}^{n}\right)\right) \rightarrow .
\end{aligned}
$$


The main result of this paper is the following generalization of Theorem A of [4].

Theorem 3.1. Let $M^{n}$ be given as in Theorem $A$ of $\S 1$. Then, the surgery map

$$
\theta:\left[M_{1}^{n} \times \mathbb{D}^{k}, \partial ; G / \text { Top }, *\right] \rightarrow L_{n+k}^{s}\left(\pi_{1} M_{1}^{n}, \omega_{1}\left(M_{1}^{n}\right)\right)
$$

is a split monomorphism provided $n+k>5$.

Just as in [4], Theorem 3.1 will be used in $\S 4$ to prove Theorem $A$ of $\S 1$ in the case $n \neq 3,4,5$ and $g: N^{n} \rightarrow M^{n}$ is a simple homotopy equivalence. To remove the restrictions of dimension and to circumvent our lack of knowledge about the Whitehead group of $\pi_{1}\left(M^{n}\right)$ we will need an addendum to the above Theorem. This will be used in $\S 4$ to prove Theorem $A$ of $\S 1$ in its complete generality.

Let $T^{3}$ denote the three-dimensional torus and consider the surgery map

$$
\theta^{\prime}:\left[M_{1}^{n} \times T^{3} \times \mathbb{D}^{k}, \partial ; G / \text { Top }, *\right] \rightarrow L_{n+k+3}^{s}\left(\pi_{1}\left(M_{1}^{n} \times T^{3}\right), \omega_{1}\left(M_{1}^{n} \times T^{3}\right)\right)
$$

which fits into an exact sequence similar to (3.1) for $\mathscr{S}\left(M_{1}^{n} \times T^{3} \times \mathbb{D}^{k}, \partial\right)$ provided $n+k+3>5$. Let

$$
\phi:\left[M_{1}^{n} \times T^{3} \times \mathbb{D}^{k}, \partial ; G / \text { Top }, *\right] \rightarrow\left[M_{1}^{n} \times T^{3} \times \mathbb{D}^{k}, M_{1}^{n} \times T^{3} \times \partial \mathbb{D}^{k} ; G / \text { Top }, *\right]
$$

denote the canonical (forgetful) map.

Addendum 3.1.1. Let $M^{n}$ be given as in Theorem $A$ of $\S 1$. Then there is a group homomorphism

$$
\begin{aligned}
& \sigma: L_{n+k+3}^{s}\left(\pi_{1}\left(M_{1}^{n} \times T^{3}\right), \omega_{1}\left(M_{1}^{n} \times T^{3}\right)\right) \\
& \quad \rightarrow\left[M_{1}^{n} \times T^{3} \times \mathbb{D}^{k}, M_{1}^{n} \times T^{3} \times \partial \mathbb{D}^{k} ; G / \text { Top }, *\right]
\end{aligned}
$$

such that the composite $\sigma \Theta^{\prime}$ is a factorization of $\phi$ provided $n+k+3>5$; i.e., $\sigma \Theta^{\prime}=\phi$.

Proof of Theorem 3.1. We shall follow the argument of [4] closely, but we shall point out the delicate point which is different from [4]. As noted in [4], because of periodicity, it suffices to show that the surgery map

$$
\theta:\left[M_{1}^{n} \times \mathbb{D}^{k} \times I, \partial ; G / T o p, *\right] \rightarrow \mathrm{L}_{n+k+1}^{s}\left(\pi_{1} M_{1}^{n}, \omega_{1}\left(M_{1}^{n}\right)\right)
$$

is split monomorphic for $k \geqq 1, n+k \geqq 5$ (where $I=[0,1]$ ). (See A.J. Nicase, Princeton thesis 1979 for the details of this result due to Quinn.) Let $x \in L_{n+k+1}^{s}\left(\pi_{1} M_{1}^{n}, \omega_{1}\left(M_{1}^{n}\right)\right)$ be represented by a surgery problem

$$
\begin{aligned}
& f:\left(W_{1}^{n+k+1} ; \partial_{+} W_{1}^{n+k+1}, \partial_{-} W_{1}^{n+k+1}, \partial_{0} W_{1}^{n+k+1}\right) \\
& \left.\quad \rightarrow\left(M_{1}^{n} \times \mathbb{D}^{k} \times I ; M_{1}^{n} \times \mathbb{D}^{k} \times 1, M_{1}^{n} \times \mathbb{D}^{k} \times 0, \partial\left(M_{1}^{n} \times \mathbb{D}^{k}\right) \times I\right)\right)
\end{aligned}
$$

satisfying the following conditions:

(i) $f_{-}: \hat{\partial}_{-.} W_{1}^{n+k+1} \rightarrow M_{1}^{n} \times \mathbb{D}^{k} \times 0$ is a homeomorphism;

(ii) $f_{+}: \partial_{+} W_{1}^{n+k+1} \rightarrow M_{1}^{n} \times \mathbb{D}^{k} \times 1$ is a simple homotopy equivalence;

(iii) $f_{0}: \partial_{0} W_{1}^{n+k+1} \rightarrow \partial\left(M_{1}^{n} \times \mathbb{D}^{k}\right) \times I$ is a homeomorphism. 
Define $U_{1}^{n+k}$ and $\partial_{ \pm} U_{1}^{n+k}$ by

$$
U_{1}^{n+k}=\partial_{+} W_{1}^{n+k+1} \quad \text { and } \quad \partial_{ \pm} U_{1}^{n+k}=f_{+}^{-1}\left(M_{1}^{n} \times \partial_{ \pm} \mathbb{D}^{k} \times 1\right)
$$

where $\partial_{+} \mathbb{D}^{k}$ and $\partial_{-} \mathbb{D}^{k}$ were defined in (2.8). Identify $\partial_{-} U_{1}^{n+1}$ with $M_{1}^{n} \times$ $\partial_{-} \mathbb{D}^{k} \times 1$ via $f_{0}$. Applying the $s$-cobordism theorem to $U_{1}^{n+k}$ with respect to $\partial_{-} U_{1}^{n+k}$ (rel. $\partial$ ), we identify $U_{1}$ as $M_{1}^{n} \times \mathbb{D}^{k}$ and we interpret $f_{+}$as a simple homotopy equivalence.

satisfying

$$
h_{1}: M_{1}^{n} \times \mathbb{D}^{k} \rightarrow M_{1}^{n} \times \mathbb{D}^{k}
$$

(i) $h_{1}^{-1}\left(\partial\left(M_{1}^{n} \times \mathbb{D}^{k}\right)\right)=\partial\left(M_{1}^{n} \times \mathbb{D}^{k}\right)$;

(ii) the restriction of $h_{1}$ to $\partial\left(M_{1}^{n} \times \mathbb{D}^{k}\right)$ is a homeomorphism;

(iii) the restriction of $h_{1}$ to $M_{1}^{n} \times \partial_{-} \mathbb{D}^{k}$ is the identity map;

(iv) the restriction $h_{1}$ to $\left(\partial M_{1}^{n}\right) \times \mathbb{I D}^{k}$ is the identity map.

Extend $h_{1}$ to $h: M^{n} \times \mathbb{D}^{k} \rightarrow M^{n} \times \mathbb{D}^{k}$ by making it the identity on $\left(M^{n}-M_{1}^{n}\right) \times$ $\mathbb{D}^{k}$.

Let us now consider the universal covers $A^{2 n+k}$ of $M^{n} \times\left(M^{n} \times \mathbb{D}^{k}\right)$ and $A_{1}^{2 n+k}$ of $M_{1}^{n} \times\left(M_{1}^{n} \times \mathbb{D}^{k}\right)$; also the universal covers $\tilde{M}^{n}$ of $M^{n}$ and $\tilde{M}_{1}^{n}$ of $M_{1}^{n}$. Let $\partial_{ \pm} A^{2 n+k}=\tilde{M} \times \tilde{M} \times \partial_{ \pm} \mathbb{D}^{k}$ and $\partial_{ \pm} A_{1}^{2 n+k}=\tilde{M}_{1} \times \tilde{M}_{1} \times \partial_{ \pm} \mathbb{D}^{k}$. Dividing out by the diagonal subgroup $\Delta n$ of $\pi \times \pi$ where $\pi$ denotes $\pi_{1} M^{n}$, we have fibrations

(i) $\tilde{M}_{1}^{n} \times \mathbb{D}^{k} \rightarrow E_{1}^{2 n+k} \rightarrow M_{1}^{n}$

(ii) $\tilde{M}^{n} \times \mathbb{D}^{k} \rightarrow E^{2 n+k} \rightarrow M^{n}$

where $E_{1}^{2 n+k}=A_{1}^{2 n+k} / \Delta \pi$ and $E^{2 n+k}=A^{2 n+k} / \Delta \pi$.

Note. (i) is a subbundle of the restriction of (ii) to $M_{1}^{n} \subseteq M^{n}$. The maps id $\times h$ and id $\times h_{1}$, respectively, induce bundle maps (again denoted by $h$ and $h_{1}$, respectively)

(i) $\tilde{M}^{n} \times \mathbb{D}^{k} \longrightarrow E^{2 n+k} \stackrel{p}{\longrightarrow} M^{n}$

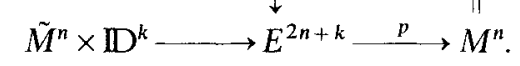

(ii) $\tilde{M}_{1}^{n} \times \mathbb{D}^{k} \longrightarrow E_{1}^{2 n+k} \stackrel{p}{\longrightarrow} M_{1}^{n}$

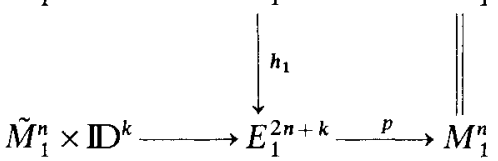

such that $h\left|\partial_{-} E^{2 n+k}=\mathrm{id}, h_{1}\right| \partial_{-} E_{1}^{2 n+k}=\mathrm{id}$ and $h\left|\partial_{+} E^{2 n+k}, h\right| \partial_{+} E_{1}^{2 n+k}$ are homeomorphisms (where $\partial_{ \pm} E^{2 n+k}$ and $\partial_{ \pm} E_{1}^{2 n+k}$ are the parts of $\partial E^{2 n+k}$ and $\partial E_{1}^{2 n+k}$ corresponding to $\partial_{ \pm} A^{2 n+k}$ and $\partial_{ \pm} A_{1}^{2 n+k}$, respectively). 
We have described in $\S 2$ [cf. 4] how the compactification of $\tilde{M}^{n}$ to $\mathbb{D}^{n}$ induces a compactification of $\tilde{M}^{n} \times \mathbb{D}^{k}$ to $S^{n-1} * \mathbb{D}^{k}$. Since the action of $\pi_{1}(M)$ on $\tilde{M} \times \mathbb{D}^{k}$ extends naturally to an action on the compactification, the fiber bundle (3.10.ii) extends to a fiber bundle

$$
S^{n-1} * \mathbb{D}^{k} \rightarrow \bar{E}^{2 n+k} \stackrel{\bar{p}}{\rightarrow} M^{n} .
$$

(See [4], p. 203, for more details.) Likewise the bundle map $h$ extends to

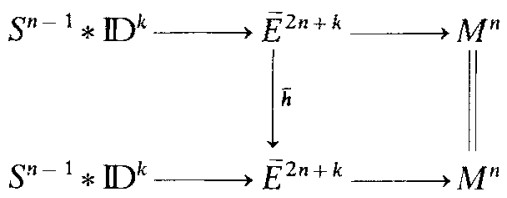

Corresponding to $S^{n-1} * \partial_{ \pm} \mathbb{D}^{k} \subseteq S^{n-1} * \mathbb{D}^{k}$, there are two subbundles of (3.13)

$$
S^{n-1} * \hat{\partial}_{ \pm} \mathbb{D}^{k} \rightarrow \partial_{ \pm} \bar{E}^{2 n+k} \stackrel{\vec{p}}{\longrightarrow} M^{n} .
$$

Each of these subbundles is left invariant by $\bar{h}$ and $\bar{h} \mid \partial_{-} \bar{E}^{2 n+k}=\mathrm{id}$ and $\bar{h} \mid \partial_{+} \bar{E}^{2 n+k}$ is a homeomorphism.

Let us now perform a fiberwise deformation of $\bar{h}$ to id. This part of the construction has no analogue in the closed case.

Let $B_{i} \times(0, \infty)(i=1,2, \ldots, q)$ be a cusp of $M^{n}$. Fix a lifting of the universal cover $\tilde{B}_{i} \times[1, \infty) \times \mathbb{D}^{k}$ of $B_{i} \times[1, \infty) \times \mathbb{D}^{k}$ to $\tilde{M}^{n} \times \mathbb{D}^{k}$ as in $\S 2$. Let $\tilde{h}: \tilde{M}^{n} \times$ $\mathbb{D}^{k} \rightarrow \tilde{M}^{n} \times \mathbb{D}^{k}$ be the "canonical" lifting of $h$ (such that $\tilde{h} \mid \tilde{M}^{n} \times \partial_{-} \mathbb{D}^{k}=\mathrm{id}$ ); also denote by $\tilde{h}$ the unique extension of this map to a self-homotopy equivalence of $S^{n-1} * \mathbb{D}^{k}$. Substituting $h$ for $g$ in (2.11), we recall that $\tilde{h}\left(\tilde{L}_{i}\right) \subset \tilde{L}_{i}$ and denote $\tilde{h} \mid \tilde{L}_{i}$ by $\tilde{h}_{i}$ which induces a homotopy equivalence

$$
h_{i}: L_{i} \rightarrow L_{i} .
$$

(Note, when $i=1$, the map $h_{1}$ in (3.15) is not the same as the $h_{1}$ in (3.8).) Now we apply Lemma 2.2 with $\tilde{h}, \tilde{h}_{i}, h_{i}$, etc. substituted for $\tilde{\mathrm{g}}, \tilde{g}_{i}, g_{i}$, etc. to obtain a deformation $H$ of $h_{i}$ to the identity map. We next show how $H$ can be used to deform $\bar{h}$ fiberwise over $B_{i} \times[1, \infty)$ to id. Abusing notation by letting $\tilde{C}_{i}=\tilde{B}_{i}$ $\times[1, \infty)$, define $\mathbb{L}_{i}=\left(\tilde{C}_{i} \times \tilde{L}_{i}\right) / \Delta\left(\pi_{1} C_{i}\right)$ and note that

$$
\tilde{L}_{i} \rightarrow \mathbb{L}_{i} \rightarrow C_{i}
$$

is a subbundle of the bundle (3.10.ii) restricted to $C_{i}$. Also, $\bar{h}$ leaves this subbundle invariant. Furthermore, $\mathbb{L}_{i}$ is an (irregular) covering space of $C_{i} \times L_{i}$ $=\left(\tilde{C}_{i} \times \tilde{L}_{i}\right) /\left(\pi_{1} C_{i} \times \pi_{1} C_{i}\right)$ and $\bar{h} \mid \mathbb{L}_{i}$ is a lift of the bundle map id $\times h_{i}$ of the bundle

$$
L_{i} \rightarrow C_{i} \times L_{i} \rightarrow C_{i} .
$$

Lift the deformation id $\times H$ to $\mathbb{I}_{i}$ to obtain a deformation $\tilde{D}_{i}$ of $h \mid \mathbb{L}_{i}$. Now define the desired deformation $D_{i}$ of $h \mid \bar{p}^{-1}\left(C_{i}\right)$ by extending $\tilde{D}_{i}$ by the identity deformation on the rest of $\bar{p}^{-1}\left(C_{i}\right)$. In particular, $D_{i}$ is a fiberwise deformation of $\bar{h} \mid \bar{p}^{-1}\left(C_{i}\right)$ to id which is constant over $\partial_{-} \bar{E}^{2 n+k} \mid C_{i}$ and over $\bar{p}^{-1}\left(C_{i}\right)-\mathbb{L}_{i}$ and is a concordance on $\partial_{+} \bar{E}^{2 n+k} \mid C_{i}$. 
After these deformations $D_{i}$ (one for each cusp $C_{i}$ where $i=1,2, \ldots, q$ ), we may assume that $\bar{h}=\mathrm{id}$ on $\bar{p}^{-1}\left(\bigcup_{i=1}^{q} B_{i} \times[1, \infty)\right)$. Now we produce a fiberwise homotopy $\widetilde{h}_{t}(0 \leqq t \leqq 1)$ of $\bar{h}$ to the identity map $\left(\bar{h}_{0}=\bar{h}, \bar{h}_{1}=\mathrm{id}\right)$ such that

(i) $\bar{h}_{t} \mid \partial \vec{E}$ is a homeomorphism (isotopy);

(ii) $\bar{h}_{t} \mid \partial_{-} \bar{E}^{\cup} \bar{p}^{-1}\left(\bigcup_{i=1}^{q} B_{i} \times[1, \infty)\right)=\mathrm{id}$;

(iii) $\bar{h}^{-1}(\partial \bar{E})=\partial \bar{E}$.

This is done by using Lemma 2.1 of [4] (Alexander's trick) since we only have to produce the homotopy (isotopy) of the fibers over the simplices outside of the cusps $\bigcup_{i=1}^{q} B_{i} \times[1, \infty)$.

We briefly recall that $\vec{h}_{t}$ is constructed inductively over $\vec{p}^{-1}\left(K^{i}\right) \quad(i=$ $0,1,2, \ldots)$ where $K^{i}$ is the $i$-skeleton of a triangulation for $M_{1}$. The inductive step is accomplished by using two facts. First, the group of homeomorphisms of $\mathbb{D}^{m}(m=0,1,2, \ldots)$ which are the identity on $\partial \mathbb{D}^{m}$ is contractible. Second, the space of continuous self-maps of $\mathbb{D}^{m}(m=0,12, \ldots)$ which restrict to the identity map on $\partial \mathrm{ID}^{m}$ is also constractible. See the proof of Lemma 2.1 of [4] for the details of the construction of $\bar{h}_{t}$. The concatenations of the deformations $D_{i}$ and the homotopy $\bar{h}_{t}$ is a careful deformation of $\bar{h}$ to id: i.e., it is a fiberwise deformation of $\bar{h}$ to id so that on $\tilde{C}_{i} \times \tilde{C}_{i} \times \mathbb{D}^{k} / \Delta \pi$ it is the identity deformation. Note that one cannot get a careful deformation from using the Alexander trick alone because the group of homeomorphisms of $\mathbb{D}^{n}$ which are the identity on both $\partial \mathbb{D}^{n}$ and a chorodisc inside of $\mathbb{I D}^{n}$ is faraway from being contractible.

We are now in the position to apply all the arguments of [4]. Let $W^{n+k+1}$ be defined by

$$
W^{n+k+1}=W_{1}^{n+k+1} \cup\left(\bigcup_{i=1}^{q} B_{i} \times[1, \infty) \times \mathbb{D}^{k} \times I\right)
$$

and extend $f$, cf. (3.5), to $f: W^{n+k+1} \rightarrow M^{n} \times \mathbb{D}^{k} \times I$ by $f(x)=x$ if

$$
x \in \bigcup_{i=1}^{q}\left(B_{i} \times[1, \infty) \times \mathbb{D}^{k} \times I\right) .
$$

Consider the universal cover $\tilde{W}^{n+k+1}$ of $W^{n+k+1}$ and define $V^{2 m+k+1}$ by

$$
V^{2 n+k+1}=\tilde{M}^{n} \times \tilde{W}^{n+k+1} / \Delta \pi .
$$

We have the following fibrations

$$
\tilde{W}^{n+k+1} \rightarrow V^{2 n+k+1} \stackrel{q}{\rightarrow} M^{n}
$$

and id $\times \tilde{f}$, where $\tilde{f}: \tilde{W} \rightarrow \tilde{M} \times \mathbb{D}^{k} \times I$ is the "canonical" lift of $f$, induces a 
bundle map

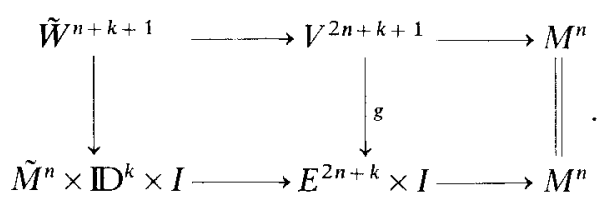

Note that we may identity the parts of $V^{2 n+k+1}$ corresponding to $\tilde{M}^{n}$ $\times \partial_{-} \tilde{W}^{2 n+k+1} / \Delta \pi$ and $\tilde{M}^{n} \times \partial_{+} \tilde{W}^{2 n+k+1} / \Delta \pi$ as $E^{2 n+k} \times 0$ and $E^{2 n+k} \times 1$, re-

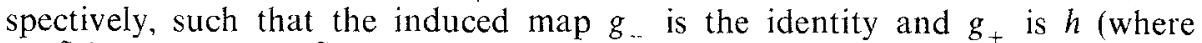
$\partial_{-} \tilde{W}^{2 n+k+1}$ and $\partial_{+} \tilde{W}^{2 n+k+1}$ are the universal covers of

and

$$
\text { o. } W_{1}^{2 n+k} \cup\left(\bigcup_{i=1}^{q} B_{i} \times[1, \infty) \times \mathbb{D}^{k} \times 0\right)
$$

$$
\partial_{+} W_{1}^{2 n+k} \cup\left(\bigcup_{i=1}^{q} B_{i} \times[1, \infty) \times \mathbb{D}^{k} \times 1\right),
$$

respectively). By attaching a careful deformation on "top of $V$ " (i.e., to $E^{2 n+h}$ $\times 1$ ), we may assume that $g_{+}=i d$ and hence that $g \mid \partial V^{2 n+k+1}$ is a homeomorphism; cf. (3.6ii). Although after attaching this deformation $g$ restricted to $q^{-1}\left(\bigcup_{i=1}^{q} C_{i}\right)$ is not the identity map (cf. $3.21,3.22$ ), it is very important to note that by (3.18) and (3.19), $g(x)=x$ for each $x \in \mathscr{K}_{i}(i=1,2, \ldots, q)$ where

$$
\mathscr{K}_{i}=\left(\tilde{C}_{i} \times \tilde{C}_{i} \times \mathbb{I D}^{k}\right) / \Delta\left(\pi_{1} C_{i}\right) \times I .
$$

(Recall that $C_{i}=B_{i} \times[1, \infty)$.)

Identify $M^{n} \times \mathbb{D}^{k} \times I$ with the "diagnal submanifold" of $E^{2 n+k} \times I$ consisting of the quotient points corresponding to points of the form $(x, x, y)$ for $x \in \tilde{M}^{n}$ and $y \in \mathbb{D}^{k} \times I$. Notice, because of the remarks in the previous paragraph, that $g$ is split along $\partial\left(M^{n} \times \mathbb{D}^{k} \times I\right)$ and along $C_{i} \times \mathbb{D}^{k} \times I$ for each $i=1,2, \ldots, q$. (Also, $g$ is a homeomorphism when restricted to the inverse images of these spaces.) Applying transversality to $g$, relative to $\partial V^{2 n+k+1} \cup\left(\bigcup_{i=1}^{q} \mathscr{K}_{i}\right)$, with respect to the "diagonal submanifold" $M^{n} \times \mathbb{D}^{k} \times I \subset E^{2 n+k} \times I$ we obtain a submanifold $N^{n+k+1}$ of $V^{2 n+k+1}$ and a degree one map from $N^{n+k+1}$ to $M^{n} \times \mathbb{D}^{k} \times I$ which is a homeomorphism when restricted to the union of $\partial N^{n+k+1}$ and the inverse image of $\bigcup_{i=1}^{q}\left(C_{i} \times \mathbb{I}^{k} \times I\right)$. So it determines an element of $\left[M_{1}^{n} \times \mathbb{D}^{k} \times I, \partial\right.$; $G /$ Top, $*]$.

To show that the element in $\left[M_{1}^{n} \times \mathbb{D}^{k} \times I, \partial ; G / T o p, *\right]$ depends only on the original surgery obstruction we take two representative normal maps $f$ : $W_{1} \rightarrow M_{1}^{n} \times \mathbb{D}^{k} \times I$ and $f^{\prime}: W_{1}^{\prime} \rightarrow M_{1}^{n} \times \mathbb{D}^{k} \times I$ which represent the same element in the surgery group. We perform the above construction to $f_{1} \mid f_{1}^{-1}\left(M_{1} \times \mathbb{D}^{k}\right.$ $\times\{1\})$ and $f_{1}^{\prime} \mid f_{1}^{\prime-1}\left(M_{1} \times \mathbb{D}^{k} \times\{1\}\right)$ to produce normal maps $g$ and $g^{\prime}$ which are homeomorphisms on the boundary. Since $f$ and $f^{\prime}$ represent the same element 
in the surgery group there is a normal bordism $F: \bar{W}_{1} \rightarrow M_{1} \times \mathbb{D}^{k} \times I \times I$ between them. We perform a relative version of the above construction to $F$. This yields a normal bordism which is a homeomorphism over $\left(M_{1} \times \mathbb{D}^{k} \times I\right)$ $\times I$ between $g$ and $g^{\prime}$. This implies that $g$ and $g^{\prime}$ determine the same element in $\left[M_{1} \times \mathbb{D}^{k} \times I, \hat{\partial} ; G /\right.$ Top, $\left.*\right]$ under the above construction.

In order to perform the construction on $F$ we need a relative version of Lemma 2.2. This relative version is proved from [6] in the same way that Lemma 2.2 is. Otherwise the details of the construction follow those in [4] closely.

Following the rest of the argument in [4], we produce, using this element, a left inverse

$$
L_{n+k+1}^{s}\left(\pi_{1} M^{n}, \omega_{1}\left(M^{n}\right)\right) \rightarrow\left[M_{1}^{n} \times \mathbb{D}^{k} \times I, \partial ; G / \text { Top }, *\right]
$$

to $\theta$.

This can be seen as follows. Let $f: W_{1} \rightarrow M_{1}^{n} \times \mathbb{D}^{k} \times I$ represent an element of $\left[M_{1}^{n} \times \mathbb{D}^{k} \times I, \partial\right.$; G/Top, $\left.*\right]$. Therefore, $f \mid \partial W_{1}: \partial W_{1} \rightarrow\left(M_{1}^{n} \times \mathbb{D}^{k} \times I\right)$ is a homeomorphism, and we do not need to deform $g$. The inverse image of the 'diagonal submanifold' $M_{1}^{n} \times \mathbb{D}^{k} \times I \subset E_{1} \times I$ can be taken to be the quotient by $\Delta \pi$ of the graph of $\tilde{f}: \tilde{W}_{1} \rightarrow \tilde{M}_{1}^{n} \times \mathbb{D}^{k} \times I$ in $\tilde{W}_{1} \times \tilde{M}_{1}^{n} \times \mathbb{D}^{k} \times I$. Hence, we get $f$ : $W_{1} \rightarrow M_{1}^{n} \times \mathbb{D}^{k} \times I$ back and we have a left inverse to $\theta$. Now suppose $f_{1}$ : $W_{1} \rightarrow M_{1}^{n} \times \mathbb{D}^{k} \times[0,1], f_{2}: W \rightarrow M_{1}^{n} \times \mathbb{D}^{k} \times[1,2]$ represent two elements $x_{1}, x_{2}$ of $L_{n+k+1}^{s}\left(\pi_{1} M^{n}, \omega_{1}\left(M^{n}\right)\right)$. Let $g_{1}, g_{2}$ be the maps of (3.22) corresponding to $f_{1}$, $f_{2}$. As we observed before, we may deform $\left(g_{1}\right)_{+}\left(g_{+}\right.$corresponding to $\left.f_{1}\right)$ to be the identity and we may stack the inverse images of diagonal submanifolds for $g_{1}, g_{2}$ together to produce the inverse image of diagonal submanifold for $x_{1}$ $+x_{2}$ of $L_{n+k+1}^{s}\left(\pi_{1} M^{n}, \omega_{1}\left(M^{n}\right)\right)$. This represents the addition of the corresponding elements of $\left[M_{1}^{n} \times \mathbb{D}^{k} \times I, \partial ; G / T o p, *\right]$, and (3.25) is a homomorphism.

In proving Addendum 3.1.1 we need to construct a function

$$
\begin{aligned}
\sigma: L_{n+k+3}^{s}\left(\pi_{1}\left(M_{1}^{n} \times T^{3}\right), \omega_{1}\left(M_{1}^{n} \times T^{3}\right)\right) \\
\quad \rightarrow\left[M_{1}^{n} \times T^{3} \times \mathbb{D}^{k}, M_{1}^{n} \times T^{3} \times \partial \mathbb{D}^{k} ; G / \text { Top }, *\right] .
\end{aligned}
$$

We begin, as before, with a normal map $f: W \rightarrow M_{1} \times T^{3} \times \mathbb{D}^{k} \times I$ representing an element in $L_{n+k+3}^{s}\left(\pi_{1}\left(M_{1}^{n} \times T^{3}\right), \omega_{1}\left(M_{1}^{n} \times T^{3}\right)\right)$. We triangulate $M_{1} \times T^{3}$ and perform the fiberwise Alexander trick in the associated bundle constructed as (3.18) (omitting condition (ii) of (3.18)). This produces a normal map which is a homeomorphism over $M_{1} \times T^{3} \times \partial\left(\mathbb{D}^{k} \times I\right)$. Argument like the ones above show that this construction determines a factorization of the forgetful map.

Notice that in the proof of Addendum 3.1.1 we make no use of delicate deformations of the maps and hence no reference to Lemma 2.2. This is fortunate because $M^{n} \times T^{3}$ only has non-positive sectional curvature and not strictly negative sectional curvature. Thus, the more delicate geometric information about the cusps needed for 2.2 is not available. The reason that we needed Lemma 2.2 in the proof of 3.1 was to make sure that the normal map between the fiber bundles constructed in (3.22) when restricted over the diagonal submanifold was a homeomorphism in the cusps. For this we needed a careful deformaton over the cusps. As noted before, this careful deformation 
must come from a direct study of the cusps since there is no version of the Alexander trick which preserves the homeomorphisms of $\mathbb{D}^{n}$ which are the identity on both $\partial \mathrm{D}^{n}$ and a horodisc inside of $\mathbb{D}^{n}$. This then is the delicate new part of the argument that one needs in addition to the ideas of [4] to prove 3.1. This study of the cusps is irrelevant however for Addendum 3.1.1.

\section{§4. Proof of Theorem A and Concluding Remarks}

Let $g: N^{n} \rightarrow M^{n}$ be a proper homotopy equivalence. Recall from [7] and [11] the notion of simple homotopy equivalence. In fact, since $W h \pi_{1} B_{i}=0$ and $\tilde{K}_{0} \mathbb{Z}\left(\pi_{1} B_{i}\right)=0$ (for $\left.i=1,2, \ldots, q\right)$, by [7], g determines an element $\tau(g) \in W h \pi_{1} M$ which vanishes if and only if $g$ is a proper simple homotopy equivalence. We conjecture that $W h \pi_{1} M=0$ but cannot prove this. In any event if $g$ is a simple equivalence and $n>5$, then Theorem A can be deduced from Theorem 3.1 and Lemma 2.1 by a method very similar to that which we gave in [4] to deduce Corollary B from Theorem A of [4]. Namely, if $g: N^{n} \rightarrow M^{n}$ is a proper simple homotopy equivalence, then we can deform $g$ until it is a homeomorphism over the cusps. Then we have a simple homotopy equivalence $g_{1}: N_{1} \rightarrow M_{1}$ which is a homeomorphism on $\partial N_{1}$. Applying 3.1 we see that $g_{1}$ is normally bordant relative to $\partial N_{1}$ to a homeomorphism. Let $G: V \rightarrow M_{1} \times I$ be such a normal bordism. Cross with $\mathbb{D}^{3}$ to get $G \times \mathrm{id}_{\mathbb{D}^{3}}: V \times \mathbb{D}^{3} \rightarrow M_{1} \times \mathbb{D}^{3} \times I$. By the $\pi$ $-\pi$ theorem [10], [12] we can do surgery on this normal map relative to $\left(M_{1}\right.$ $\left.\times \mathbb{D}^{3} \times \partial I\right) \cup\left(\partial M_{1} \times \mathbb{D}^{3} \times I\right)$ to make it a simple homotopy equivalence. It then becomes an $s$-cobordism and therefore a product between $N_{1} \times \mathbb{D}^{3}$ and $M_{1} \times$ $\mathbb{D}^{3}$. It provides a homotopy from $g_{1} \times \mathrm{id}_{\mathbb{D}^{3}}$ to a homeomorphism. This homotopy is an isotopy over $\partial M_{1} \times \mathbb{D}^{3}$. This proves that $g \times \mathrm{id}_{\mathbb{D}^{3}}$ is homotopic to a homeomorphism relative to $\partial M_{1} \times \mathbb{D}^{3}$. Such a homotopy of course provides a proper homotopy of $g \times \mathrm{id}_{\mathbb{R}^{3}}: N \times \mathbb{R}^{3} \rightarrow M \times \mathbb{R}^{3}$ to a homeomorphism.

Unfortunately, for this argument we assumed that $n \geqq 6$ and that $g$ was a simple homotopy equivalence. Now we give another argument using Addendum 3.1.1 instead of Theorem 3.1. It does not have these restrictions on $g$ and $n$ but it will produce a proper homotopy of $g \times \mathrm{id}_{\mathbb{R}^{3}}$ to a homeomorphism which is uncontrolled in the cusps.

Notice that we may assume that $n>2$ without loss of generality since Theorem A is trivial if $n \leqq 2$. By the product formula of [11], $g \times$ id: $N^{n}$ $\times T^{3} \rightarrow M^{n} \times T^{3}$ is a simple homotopy equivalence. By Addendum 2.1.1, $\mathrm{g} \times$ id is homotopic to $h: N^{n} \times T^{3} \rightarrow M^{n} \times T^{3}$ such that $h$ is a homeomorphism when we restrict $h$ to $h^{-1}\left(C_{i} \times T^{3}\right)$ for $i=1,2, \ldots, q$. (This is not valid for locally symmmetric spaces of higher rank.) Let $h_{1}: N_{1}^{n+3} \rightarrow M_{1}^{n} \times T^{3}$ denote the restriction of $h$ to $N_{1}^{n+3}=h^{-1}\left(M_{1}^{n} \times T^{3}\right)$ where $M_{1}^{n}$ is defined by formula (2.7). Note that $h_{1}$ is a simple homotopy equivalence and the restriction of $h_{1}$ to $\partial N_{1}$ is a homeomorphism. Hence, $h_{1}$ determines an element $\left[h_{1}\right] \in \mathscr{S}\left(M_{1}^{n} \times T^{3}, \partial\right)$. Consider the canonical "forgetful" map

$$
\psi: \mathscr{S}\left(M_{1}^{n} \times T^{3}, \partial\right) \rightarrow \mathscr{S}\left(M_{1}^{n} \times T^{3}\right)
$$


If we push $\left[h_{1}\right]$ down two steps in the surgery exact sequence for $\mathscr{S}\left(M_{1}^{n} \times T^{3}\right)$, we (of course) land at the zero element in $L_{n+3}^{s}\left(\pi_{1}\left(M_{1}^{n} \times T^{3}\right), \omega_{1}\left(M_{1}^{n} \times T^{3}\right)\right)$. Hence by Addendum 3.1.1 $\phi\left(\eta\left(\left[h_{1}\right]\right)\right)=0$ where

$$
\eta: \mathscr{S}\left(M_{1}^{n} \times T^{3}, \partial\right) \rightarrow\left[M_{1}^{n} \times T^{3}, \partial ; G / \text { Top }, *\right]
$$

is the canonical map in the surgery exact sequence for $\mathscr{S}\left(M_{1}^{n} \times T^{3}, \partial\right)$. But the forgetful maps commute with $\eta$; i.e., $\phi \eta=\eta^{\prime} \psi$ where

$$
\eta^{\prime}: \mathscr{P}\left(M_{1}^{n} \times T^{3}\right) \rightarrow\left[M_{1}^{n} \times T^{3} ; G / \text { Top }\right]
$$

is the canonical map in the surgery exact sequence for $\mathscr{S}\left(M_{1}^{n} \times T^{3}\right)$; consequently, $\eta^{\prime} \psi\left(\left[h_{1}\right]\right)=0$.

Therefore by the exactness of the surgery exact sequence for $\mathscr{S}\left(M_{1}^{n} \times T^{3}\right)$, $N_{1}^{n+3}$ is normally cobordant to $M_{1}^{n} \times T^{3}$ (but not by keeping the boundary "fixed"). Hence $N_{1}^{n+3}-\partial N_{1}^{n+3}$ is properly normally cobordant to $M_{1}^{n} \times T^{3}$ $-\left(\partial M_{1}^{n}\right) \times T^{3}$. But, $N_{1}^{n+3}-\partial N_{1}^{n+3}$ is homeomorphic to $N^{n} \times T^{3}$ and $M_{1}^{n} \times T^{3}$ $-\left(\partial M_{1}^{n}\right) \times T^{3}$ is homeomorphic to $M^{n} \times T^{3}$. Therefore, the covering space of this normal cobordism corresponding to the subgroup $\pi_{1} M^{n}$ of $\pi_{1}\left(M^{n} \times T^{3}\right)$ is a proper normal cobordism between $N^{n} \times \mathbb{R}^{3}$ and $M^{n} \times \mathbb{R}^{3}$. Since the fundamental group of the end of $M^{n} \times \mathbb{R}^{3}$ maps isomorphically to $\pi_{1}\left(M^{n} \times \mathbb{R}^{3}\right)$, the proper $\pi-\pi$ theorem [10], [12] gives that $N^{n} \times \mathbb{R}^{3}$ and $M^{n} \times \mathbb{R}^{3}$ are properly s-cobordant and hence homeomorphic by [7], [11]. Also, it is easily seen that this homeomorphism is properly homotopic to $g \times$ id. This completes the proof of Theorem A.

We next note that Theorem A cannot be naively extended to spaces $N^{n}$ of non-positive sectional curvatures; i.e., in the statement of Theorem $A$, the words "strictly negative and bounded away from 0 and $-\infty$ " cannot be replaced by non-positive and bounded away from $-\infty$. For example, let $\Gamma_{m} \subseteq S L_{m}(\mathbb{Z})$ be a torsion-free subgroup of finite index and $N^{n}$ be the double coset space defined by

$$
N^{n}=\Gamma_{m} \backslash S L_{m}(\mathbb{R}) / S O(m)
$$

where $n=\frac{1}{2}\left(m^{2}+m-2\right)$. Then $N^{n}$ is complete, has finite volume, and nonpositive sectional curvatures which are bounded away from $-\infty$. But when $m$ is sufficiently large ( $m>200$ is adequate), there are many manifolds $M^{n}$ properly homotopically equivalent to $N^{n}$ but not even stably homeomorphic to $N^{n}$.

We may compactify $N^{n}$ to $N_{1}^{n}$ [2] such that $\pi_{1} N_{1}^{n} \simeq \pi_{1} \partial N_{1}^{n}$, i.e., $N^{n}=\operatorname{Int} N_{1}^{n}$, provided $m>3$. Choosing $m>200$, because of the stable calculation [1] of $H^{*}\left(\Gamma_{m}, \mathbb{Q}\right)$, we can let $f_{1}: M_{1} \rightarrow N_{1}$ represent an element of $\left[N_{1}, G /\right.$ Top $]$ such that its characteristic class in $H^{44}\left(N_{1} ; \mathbb{Q}\right)$ is $\neq 2 p_{11}\left(N_{1}\right)$ and 0 . Since $\pi_{1} \partial N_{1} \simeq \pi_{1} N_{1}$, it follows from $\pi-\pi$ theorem that we may assume that $f_{1}$ is a homotopy equivalence. Let $M=\operatorname{Int} M_{1}$ and $f=f_{1} \mid M: M \rightarrow N$ is a proper homotopy equivalence. But $f \times$ id: $M^{n} \times \mathbb{R}^{k} \rightarrow N^{n} \times \mathbb{R}^{k}$ is never homotopic to a homeomorphism for any $k$, because $M^{n} \times R^{k}$ and $N^{n} \times R^{k}$ have "different" rational Pontriajn classes.

But the following conjectures are plausible. 
Conjecture 4.1. Let $M^{n}$ be a compact aspherical manifold with possibly non-empty boundary. Then the surgery map

$$
\theta:\left[M^{n} \times \mathbb{D}^{k}, \partial\left(M^{n} \times \mathbb{D}^{k}\right) ; G / \text { Top }, *\right] \rightarrow L_{n+k}^{s}\left(\pi_{1} M^{n}, \omega_{1}\left(M^{n}\right)\right)
$$

is a split monomorphism provided $n+k \geqq 5$.

In this paper, we verified the above conjecture for pinched negatively curved manifolds of finite volume. (We cut off the cusps to produce the manifold with boundary.) The argument was rather delicate because we had to control the behavior of the cusps. We hope our technique will prove the conjecture for the arithemetic group case, but the control of the Borel-Serre boundary has to be much more careful.

Conjecture 4.2. With the same hypotheses as in Conjecture 4.1, the rational surgery map

$$
\theta \otimes \mathrm{id}:\left[M^{n} \times \mathbb{D}^{k}, \partial\left(M^{n} \times \mathbb{D}^{k}\right) ; G / \mathrm{Top}, *\right] \otimes \mathbb{Q} \rightarrow L_{n+k}^{s}\left(\pi_{1} M^{n}, \omega_{1}\left(M^{n}\right)\right) \otimes \mathbb{Q}
$$

is a monomorphism provided $n+k \geqq 5$.

This is Conjecture 1.2 of [4] replacing the closed aspherical manifold by a compact aspherical manifold with boundary. If one can prove this conjecture for $M^{n}$, then the so-called Novikov's conjecture (Conjecture 1.1 of [4]) is verified for the group $\pi=\pi_{1} M^{n}$. If one is not careful about $\partial M^{n}$, it is possible to think that the full Novikov's conjecture is verified when one has only shown that the kernel of $\theta \otimes$ id is contained in the kernel of the canonical "forgetful" homomorphism

$$
\phi:\left[M^{n} \times \mathbb{D}^{k}, \partial\left(M^{n} \times \mathbb{D}^{k}\right) ; G / T o p, *\right] \otimes \mathbb{Q} \rightarrow\left[M^{n} \times \mathbb{D}^{k} ; G / \text { Top }\right] \otimes \mathbb{Q}
$$

(cf. Addendum 3.1). As pointed out in [9], this is probably the reason why Miscenko at times claimed that he has proved the full Novikov's conjecture for $M^{n}$ a non-positively curved complete Riemannian manifold.

\section{References}

1. Borel, A.: Stable real cohomology of atithmetic groups. Ann. Sci. École Norm. Sup. 7, 235-272 (1974)

2. Borel, A., Serre, J-P.: Corners and arithmetic groups. Comment. Math. Helv. 48, 436-491 (1973)

3. Eberlein, P.: Lattices in spaces of nonpositive curvature. Annals of Math. 111, 435-476 (1980)

4. Farrell, F.T., Hsiang, W.C.: On Novikov's conjecture for non-positively curved manifolds, I. Annals of Math. 113, 199-209 (1981)

5. Farrell, F.T., Hsiang, W.C.: The Whitehead group of poly-(finite or cyclic) groups. J. London Math. (2) 24, 308-324 (1981)

6. Farrell, F.T., Hsiang, W.C.: Topological characterization of flat and almost flat Riemannian manifolds $M^{n}(n \neq 3,4)$. Amer. J. Math. in press (1982)

7. Farrell, F.T., Wagoner, J.P.: Algebraic torsion for infinite simple homotopy types. Comment. Math. Helv. 47, 502-513 (1972)

8. Gromov, M.; Manifolds of negative curvature. J. Diff. Geom. 13, 223-230 (1978)

9. Hsiang, W.C., Rees, H.: Miscenko's work on Novikov's conjecture. in press (1982) 
10. Maumary, S.: Proper surgery groups and Wall-Novikov groups. In: Algebraic $K$-theory III, Lecture Notes in Math. Vol. 343, pp. 526-539. Berlin-Heidelberg-New York: Springer 1973

11. Siebenmann, L.C.: Infinite simple homotopy types. Indag. Math. 32, 479-495 (1970)

12. Tayior, L.: Ph.d. thesis, Univ. of Calif., Berkeley, 1971

13. Kirby, R.C., Siebenmann, L.C.: Foundational Essays on Topological manifolds, smoothing and triangulations. Ann. of Math. Studies 88, Princeton Univ. Press, 1977

14. Wall, C.T.C.: Surgery on Compact Manifolds. New York: Academic Press 1970

Oblatum 26-V-1981 \& II-1982 